\title{
Téoros
}

Revue de recherche en tourisme

\section{La gestion déléguée des activités et services du parc d'Oka : l'expérience positive d'un partenariat avec le milieu}

\section{Martin Soucy}

Volume 14, numéro 1, printemps 1995

Les parcs : des réseaux en mutation

URI : https://id.erudit.org/iderudit/1077051ar

DOI : https://doi.org/10.7202/1077051ar

Aller au sommaire du numéro

Éditeur(s)

Université du Québec à Montréal

ISSN

0712-8657 (imprimé)

1923-2705 (numérique)

Découvrir la revue

Citer cet article

Soucy, M. (1995). La gestion déléguée des activités et services du parc d’Oka :

l'expérience positive d'un partenariat avec le milieu. Téoros, 14(1), 20-23.

https://doi.org/10.7202/1077051ar 


\section{La gestion déléguée des activités et services du parc d'Oka ${ }^{(1)}$ : I'expérience positive d'un partenariat avec le milieu \\ Martin Soucy}

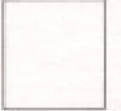

Il $y$ a maintenant cent ans le premier parcquébécois woyait le jour. Depuiscetemps, un réseau composédedix-sept territoiresa été formé, une Lai sur les parcs a été adoptée et une politique a été instituée.

Témoins d'une volonté de protéger et de mettre en valeur le patrimoine naturel du Québec, le réseau des parcs permet à la population de découvrir cette richesse tout en la conservant pour les générations futures.

Dansl'optique «d'associer les Québécoises et les Québécois au développement et à la mise en valeur des parcs», le gouvernement duQuébecdécida, dans les années 1980, de déléguer la gestion de certaines activités et de certains services, tout en demeurant propriétaire exclusif des territoires ${ }^{(2)}$. Cette initätive allait naturellement créer une nouvelle dynamique qui façonne présentement la réalité quotidienne de certains parcs québécois.

Nous croyons donc qu'il soit intéressant d'aborder cette situation en présentant l'expérience positive d'une Corporation qui participe activement, à titre de partenaire du ministère de l'Environnement et de la Faune $(\mathrm{MEF})^{0)}$, à la gestion et au développement du parc d'Oka.

Dans un premier temps, nous introduirons brièvement le site et son historique de délégation. Nous explorerons, ensuite, les divers éléments reliés à la Corporation, les Services récréatifsdu pare d'Oka (SERPO). Enfin, nous présenterons les résultats qu'elle a obtenus depuis le début de son mandat.

\section{Le parc d'Oka}

L'aménagement du parc de récréation d'Oka, tel qu'il est connu aujourd'hui, s'est fait d'une façon progressive sur une pé-

- Monsieur Martin Soucy est directeur général des Services recréatifs du pare d'Oka (SERPO) et btudiant au programme de MSc de l"Ecole des Hautes Études Commerciales de Montréal.

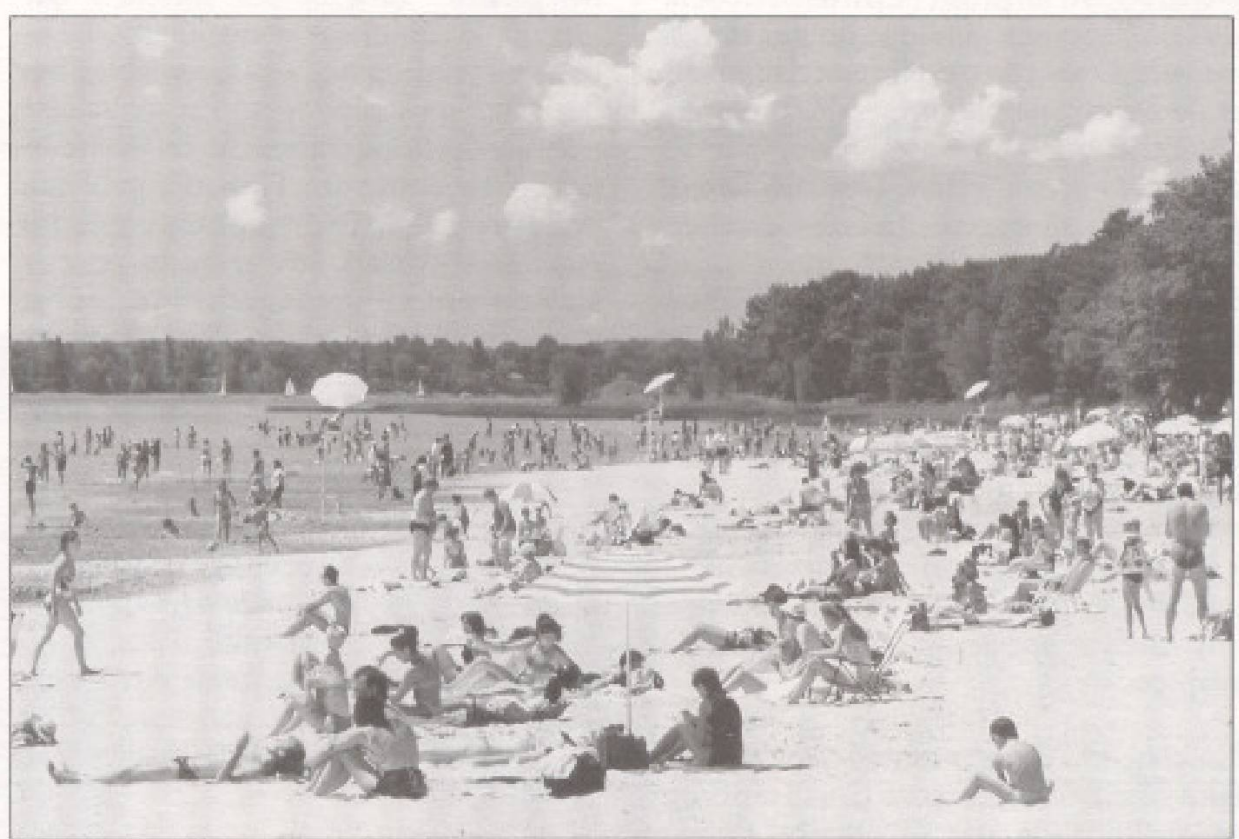

Photo: Sylvain Majenu, ministère de l'Environnement et de la Faune.

riode de 25 ans $^{(4)}$. En effet, c'est en 1962 que le Gouvernement du Québec acquiert la première parcelle de terrain, qui deviendra l'assise de ce territoire. Quelques années plus tard, les premières infrastructures de récréation y seront aménagées et serviront à accueillir les divers touristes attendus pour l'exposition universelle de 1967.

En 1974, le gouvernement consolida le territoire en se portant acquéreur de la colline du Calvaire, de la colline Masson et de la Grande Baie. Ces secteurs permettent actuellement de conserver et de mettre en valeur un patrimoine québécois à la fois naturel et culturel.

Le pare d'Oka, longtemps connu sous le nom du «parc Paul Sauvés, aura conservé un statut de réserve de chasse et de pêche, avant d'ëtre légalement constitué, au sens de la Loi sur les parcs, en 1990.

Ayant pour objectif, à titre de parc de récréation, de afavoriser la pratique d'une variété d'activités récréatives de plein air tout en protégeant l'environnement naturels s.5), ce site, situé à moins de $50 \mathrm{~km}$ de
Montréal, bénéficie d'une situation géographique particulière qui le place à proximité d'une agglomération où l'on dénombre plus de 2,6 millions d'habitants.

Sur les bords du lac des Deux-Montagnes, plage, forêt, prairies, marécages, collines et faunecomposent levisage naturel diversifié du parc d'Oka et se combinent à ses divers aménagements récréatifs d'importance pour lui permettre de répondre aux besoins des citadins qui désirent renouer avec la nature.

\section{La délégation de gestion}

Ayant assuré de façon exclusive la gestion du parc d'Oka depuis sa fondation, c'est au printemps 1986 que le ministère du Loisir, de la Chasse et de la Pếche de l'époque déléguait, à l'entreprise privée, l'exploitation des activités récréatives d'importance ainsi que les services commerciaux offerts au parc d'Oka (camping, baignade, ski de randonnée, services alimentaires, dépannage, boutique de souvenirs, etc.). Cette opération avait pour objectifs de réduireles budgets publics d'opération des parcs, tout en augmentant le nombre et la qualité des 
services offerts dans le respect de la wocation des territoires .

Deux firmes à but lucratif, devant verser une redevance sous la forme d'un loyer au Gouvernement du Québec, ont assumé le mandat avant l'arrivée de la SERPO (une Corporation sans but lucratif).

La Société de Développement Multidisciplinaire (SODEM), une entreprisespécialisée, entreautre, dansla gestion d'équipements de loisir, fut la premièré entreprise à prendre en charge le mandat au parc d'Oka. De 1986 à 1990, on enregistrera une augmentation appréciable de l'achalandage du site qui franchit même, à certeépoque, le seuil du million d'usagers'?

À l'expiration du contrat de concession de SODEM, la firme immobilière SPHINX Inc. obtient, par voie d'appel d'offres, la gestion des activités déléguées du parc d'Oka et ce, pour une période de cinq ans. Cetteentreprisenesera en faiten posteque du printemps 1990 à l'hiver 1992.

Une étuderéalisée en 1992, pour lecompte du ministère du Loisir, de la Chasse et de la Pêche ${ }^{(8)}$, démontre que le passage de cette entreprise a eu des effets négatifs sur le parc. Ayant offert des redevances jugées *irréalistes» par rapport aux revenus qu'il est possible de gênérer de l'exploitation normale des activités offertes, certe société a dû *utiliser des movens qui peuvent être caractérisés comme en dehors de la vocition d'un parcos ${ }^{(9)}$ pour dégager les sources de revenus nécessaires à la rencontre de ses obligations contractuelles. $\Pi$ appert donc, selon ce rapport, que:

- d'image et le positionnement du parc ont été changés d'un parc de récréntion "naturel" avec une clientèle largement familiale à un parc qui attire une clientèle de jeunes et d'adolescentsw;

- «plasieurs équipements ont été mal entretenus et se sont détériorésw;

- kle Ministère n'a pas perçu les revenus promis».

\section{Le début de la décennie}

D'autres éléments sont venus perturber le pare d'Oka au début de la décennie. Il faut se rappeler que la région d'Oka avait été durement touchée parla criseamérindienne de 1990. Quoique ses effets soient presque totalement effacés en 1995, grâce aux efforts concertés de plusjeurs intervenants, l'achalandage avait dramatiquement chuté à l'époque.

Enfin, avec sa fréquentation annuelle de plus de 800000 usagers, le parc d'Oka est l'un des sites les plus fréquentés du réseau des parcs et $\propto$, malgré sa superficie restreinte de $23,7 \mathrm{~km}^{2}$. En fonction de son utilisation intensive, certaines zones montrent «des signes évidents de dégradation qui risquent de compromettre la vocation du territoires $x^{(0)}$. Plusieurs sommes d'argentdevront d'ailleurs yêtre investies pour remédier à la dégradation du milieu, des équipements et des infrastructures.

C'est donc sur la base de cette toile de fond que la SERPO allait faire son entrée au parc d'Oka avec la ferme intention d'aider le $M E F$ à remédier à la situation.

\section{Les services récréatiffs du parc d'Oka}

Le projet d'une gestion locale pour le parc d'Oka, et d'un partenariat aveclesautorités gouvernementales impliquées, remonte à la fin desannées 1980. En effet, leMaire de la Paroisse d'Oka avait signifié dès 1988 , au ministre du Loisir, de la Chasse et de la Pêche, l'intention des intervenants locaux de prendre en main la gestion des activités déléguées de ce joyau provincial. Il aura fallu plusieurs interventions des instances locales et près de quatre ans avant que, le 5 juin 1992, laSERPO débute sesopérations.

Prenant ainsi la relève des deux firmes privées, une Corporation à but non lucratif dont la formation a été parrainée par les municipalités de la Paroisse et du Village $\mathrm{d}^{7} \mathrm{Oka}$, devenait la première entreprise du genre à assumer la gestion et le développement des activités récréotouristiques du parc d'Oka.

\section{Le partenariat avec le MEF}

Liếe au Gouvernement du Québec par le biais d'un contrat d'autorisation, qui lui assure que la Corporation offrira des activités et des services de qualité dans le respect des lois et règlements en vigueur (dont la Loi sur les parcs), la SERPO est responsable de la gestion de la zone récréative qui représente environ $11 \%$ de l'ensemble du territoire du parc d'Oka.
En plus de superviser les actions de la Corporation, le MEF est quant à lui responsable des zones de préservation, d'ambiance et de services. Il veille à assumer lesmissions de protection du territoire, d'accueil des usagers et d'éducation au milieu naturel.

Dès son arrivée en 1992, la SERPO a entretenu de saines relations avec les autorités du Ministère en place au parc d'Oka. Partageant des objectifs communs, soient ceux d'offrir des services de qualité aux usagers et de favoriser la protection du parc d'Öka, nous croyons avoir développé un partenariat intéressant qui permet de bien servir les intérêts du territoireet de ses divers visiteurs.

Par exemple, par le biais de rencontres régulières de coordination, nous cultivons une relation de confiance qui nous permet d'aborder et de solutionner conjointement les problématiques de gestion. Nous favorisons de plus les échanges de services dans certains domaines et nous avons réalisé dernièrement ensemble des travaux d'immobilisation.

Outre ce partenariat profitable avec les autorités gouvernementales, l'arrivée de la SERPO allait modifier la gestion des activités déléguées en leur faisant prendre un virage important et nécessaire. A titre d'exemple, on peut citer les changements suivants :

- D'abord, la SERPO s'est engagée à réimestir la totalité de ses revenus nets dans le parc d' $\mathrm{Oka}$, faisant ainsi toute la différence avecles entreprises quil'ont précédée. En effet, les profits qui sont générés desactivités déléguées servent entièrement et directement le parcen permettant del'améliorer et d'en faire bénéficier immédiatementlesusagers.

- La SERPO s'est de plus donnée la priorité d'attendre l'excellence dans l'offre desactivités et des servicesdont elle a la charge, tout en maintenant la rentabilité de ses opérations. L'orientation de gestion de la Corporation repose done sur l'atteinte d'un juste équilibre entre ces deux variables importantes, le tout placé dans l'optique des missions premières du pare d'Oka.

Il n'est donc pas question de réduire la qualité des services pour obtenir un maximum de profit, pas plus que de faire preuve 
d'une mauvaisegestion budgétaire pour ne couvrir que les dépenses ou faire un déficit. Selon les orientations de la SERPO, la notion d'entreprise sins but lucratif n'est pas synonyme de non rentabilité. Au contraire, la Corporation se doit d'être aussi efficace, sinon plus, qu'une entreprise privée à but lucratif touten ayant unevision qui répond davantage à la réalité des parcs provinciaux. Les administrateurs et la direction de la SERPO sont donc conscients des besoins du parc d'Oka et ils ont la responsabilité d'agir en fonction des intérêts de ce territoire.

\section{L'implication du milieu et des usagers}

Un autre élément majeur différencie la SERPO des autres entreprises qui ont oeuvré au parc d'Oka: le contrôle et les orientations de l'organisme qui a la charge des activités déléguées du parc d'Oka sont maintenant sous la gouverne des gens du milieu.

Ces derniers sont, selon nous, les plus près des besoins de leur communaté et les plus sensiblesà l'importance de bien servir et de bien protéger ce territoire dont ils s'enorgueillissent. Dans les faits, cela se traduit de la façon suivante.

La Corporation compte actuellement cing membres en règle qui forment le conseil d'administration: deux membres sont nommés par la paroisse d'Oka, deux par le village d'Oka et un par la Chambre de commerce d'Ok: ${ }^{(11)}$. A titre bénévole, les administrateurs ont la responsabilité d'établir, de concert avec la direction, les grandes orientations et de veiller à la bonne gestion du mandat qui a été confié à la SERPO,

De plus, dans l'optique d'impliquer activement les usagers dans la gestion de la Corporation et du pare d'Oka, deux sièges leur seront offerts au sein du conseil d'administration à compter de $1995^{(12)}$. La SERPO impliquera ainsi celles et ceux que l'on sert chaque jour et qui sont en fait une très grandesource d'information pour tous.

Nous favorisons enfin l'implication de divers regroupements spécialisés régionaux. Par le biais de comités consultatifs, ces derniers sont appelés à s'impliquer activement dans certains dossiers touchant le parc d'Oka et ce, en fonction de leur mission respective. Cette initiative récente permettra certainement de développer un plus grand sentiment d'appartenance régional à l'égard du parc d'Oka.

\section{Les résultats}

S'étant donnée comme priorité de redorer l'imagedu parc d'Oka, et favoriser le retour d'une clientèle familiale, la SERPO s'est efforcéed'appliquer unestratégie derelance basée sur les éléments suivants:

- miser sur le travail d'équipe et sur une écoute active des besoins des usagers;

- améliorer significativement la qualité des activités et des services offerts, tant au niveau du travail quotidien que du développement de nouveaux produits;

- promouvoir le territoire et ses diverses activités:

- Gavoriser la restauration et la protection du parc d’Oka.

Plusieurs variables nous permettent d'évaluer le rendement de la Corporation et les résultats que nous présentons s'attarderont à divers éléments significatifs.

\section{Les usagers}

En fonction des conditions climatiques et des autres facteurs que nous avons exposés précédemment, la fréquentation des activités, dont la SERPO a la responsabilité, connaissait un très bas niveau en 1992 . Depuis lors, on note une augmentation significative de l'achalandage et ce, à tous les niveaux.

L'amélioration la plus importante est par exemple enregistrée au nivesu de la fréquentation du camping. En effet, nous avons noté une augmentation de près de 54 \% de la location d'emplacements de camping (celle-ci passant de 13954, en 1992, à 21511 au 31 août 1994) ${ }^{(19)}$. Nous avons d'ailleurs franchi le seuil des 700 emplacements loués (sur une possibilité de 800) à trois reprises au cours de la sison 1994, ce qui n'avait pas été vu depuis 1989.

Sur la base de nos observations, nous avons de plus constaté une modification du profil de la clientèle. La grande majorité des usagers se compose maintenant de familles et de retraités et ce, contrairement à 1991 = 1992 ou les adolescents et les jeunes avaient pris d'assaut le parc.
Enfin, d'un point de vue général, le niveau des plaintes a diminué significativement depuisl'arrivée delaSERPO. Leur nombre est en effet passé d'une quinzaine par semaine en 1991, à un peu moins de quatre par mois aujourd'hui. Les commentaires positifs des clientsetduMEF sontd'ailleurs une grande source de motivation pour les membres de l'Équipe-SERPO.

\section{Les mentions touristiques}

Le rendement de la Corporation peut également être souligné sur la base de mentions touristiques. C'est ainsi qu'elle s'estméritée deux prixd'importance depuis sa création.

Le premier, celui de l'Ambassadeur du meilleur accueil touristique, décerné en 1993, par la Corporation de Développement Touristique de Deux-Montagnes, est venu souligner les efforts de la nouvelle équipe à la suite de la première saison d'exploitation.

Au printemps 1994, dans le cadre des Grands Prix du Tourisme Québécois, l'Association Touristique des Laurentides décernait la seconde mentiond'importance pour l'entreprise. En effet, grâce à son concéptde forfaits-camping wclés en main», la SERPO remportait le lauréat régional dans la catégorie innovation touristique.

\section{Les retombées locales}

Désirants'impliquer dans la communauté, la SERPO a participé à plusieurs événements pour venir en aide aux organismes oeuvrant dans la région. Que ce soit par la présentation, en collaboration avec les Clubs Optimistes, d'une Fête Champêtre sur l'environnement ou par l'entremise de commandites d'événements régionaux et d'organismes à but non-lucratif $f^{(14)}$, la SERPO a été un citoyen corporatif très impliquédepuis son arrivée. Ellesupporte de plus une équipe de coureurs à pied d'Oka, qui participe au Marathon de Montréal, et une équipe de jeunes sauveteurs.

Au chapitre de l'emploi, la SERPO embauche annuellement près de 85 employés, dont la majorité sont des étudiants, et plus de $80 \%$ proviennent de la région d'Oka. Au 31 mars 1995, soit après ses trois premières années d'exploitation, la SERPO 
aura versé près de 1,3 million de dollars en salaires dans la région.

D'autres retombées économiques importantes sont également à noter en fonction des politiques d'achat local de la Corporation dont bénéficient les commerçants d'Oka et de la MRC de Deux-Montagnes. À cet effet, les sommes destinées à l'achat de biens etde services locaux aurontexcédé les $850000 \$$ au 31 mars 1995 .

\section{Les réinvestissements en immobilisation}

Comme nous l'avons mentionné précédemment, la totalité desprofitsnets générés par la Corporation doivent être réinvestis dans les immobilisations du parc d'Oka. Ainsi, au terme de sa troisième année d'exploitation, et selon nos prévisions ${ }^{(15)}$, la SERPO devrait avoir investi une somme de près de $187000 \mathrm{~S}$ à ce chapitre. Les projets réalisés sont notamment:

- l'aménagement d'un centre administratif, plus accessible à la clientèle, ainsi que d'un nouveau local pour le dépanneur qui nous a permis de libérer une salle complète pour les activités d'animation;

- l'aménagementd'un parcpourenfant au centre communautaire et d'une nouvelle barrière;

- des rénovations mineures de l'accueil camping, de la buanderie, du système de douches extérieures à la plage et du système de plomberie dans certaines unités sanitaires du camping;

- l'achat de tables à pique-nique et la réparation d'une route sur la plage;

- et, la réparation du système d'égout tertiaire d'un secteur de camping.

Combinés aux autres éléments du bilan, ces résultats sont selon nous très positifs et ce, compte tenu d'un léger déficit d'opération enregistréla première année ${ }^{110}$, des différentes difficultés rencontréesen début de mandat et de la condition générale des équipements du parc d'Oka.

Afin de soutenir les efforts du MEF, la Corporation a créé un fonds d'investissement en environnement. Le FONDSSERPO générera ainsi, au cours des prochaines années, des sommes d'argent provenant de sources externes aux opérations régulières du parc d'Oka. Ces dernières permettront au MEF de réaliser des pro- jets environnementaux liés au territoire telsle parachèvement d'un sentier pédestre ou la reforestation de certaines zones problématiques.

La Corporation a également lancé à l'été 1994, un programme de recyclage des déchets s'adressant aux campeurs, ce qui fut une première dans un parc provincial.

\section{Conclusion}

L'expérience que nous avons présentée démontre clairement que les intervenants locaux sont en mesure de participer activement, à titre de partenaire du MEF, à la gestion et au développement des pares québécois.

Nous avons également la certitude qu'il existe, danschacune des régionsdu Québec, des ressources qui ne demandent qu'à s'impliquer dans l'avancement du devenir des pares en mettant à contribution leur dynamisme et leur savoir-faire.

En considérant l'étar des finances publiques, nous croyons qu'il serait intéressant pour le Gouvernement du Québec de s'adjoindre ces partenaires pour une durée de plus de cinqans. Cetteinitiative permettrait, d'une part, de s'assurer d'un financement privé à long terme indispensable à l'amélioration de chacun des territoires. D'autre part, elle favoriserait une stabilitédans la gestion des activités dáléguées, en maintenant en place lespartenaires qui répondent adéquatement aux besoins et aux objectifs des parcs québécois.

En fonction de l'expertise qu'elle aura développée, de l'expérience qu'elle aura acquise, de ses nombreuses réalisations et de sa volonté de préserver le parc d'Oka, la SERPO espère bien, en partenariat avec le MEF, avoirl'opportunité d'oeuvrer encore plusieurs années au parc d'Oka et ce, afin d'assurer la continuité indispensable des actions entreprises dans l'intérêt ultime du territoire et des usagers qui le fréquentent.

\section{RÉFÉRENCES}

(1) Le parc d'Oka est une proprieté du Gouvernement du Québec dont cortaines activités sont gérées par la SEAPO, un organisme sans but lucratif.

(2) Laliberté, Lanctót, Coopers \& L Lybrand, Évaluation de la délégation de gestion: rapport final, ministère du Loisir, de la Chasse et de la Péche, 31 mars 1992, p. 5
(3) Depuis la fusion du ministère de l'Environnement et de celui du Loisir, de la Chasse et de la Pëche et ci-après nommé MEF,

(4) Ministere du Loisir, de la Chasse et de la Peche, Pare Paul Sauve: plan dírecteur d'aménagement, mars 1981, pp. 9-42.

(5) Ministere du Loisir, de la Chasse et de la Péche. Les parcs québecols: la politique, avril 1982, p. 65.

(6) Laliberte, Lanctôt, Coopers \& Lybrand, op. cit., p. 9

(7) Ministêre du Loisir, de la Chasse et de la Pèche, Statistiques compilés par le parc d'Oka.

(B) Laliberté, Lanctôt, Coopers \& Lyorand, op. cit.. p. 15.

(9) Fetes diverses commanditées, utilisation abusive de distributrices, vente d'articles promotionnels né répondant pas à l'image des parcs, installations de jeux video, etc.

(10) Ministère du Loisir, de la Chassse et de la Péche, Plan des bquipements du parc d'Oka, document présenté conjointement par la Direction du plein air et des parcs et la Direction rógionale de Montréal décembre 1990, p. 6

111) Il est egalement important de spécifier que légalement, la Corporation est independante des deux. entites municipales et de la Chambre de commerce.

(12) La création de ses deux nouveaux sièges ne modifie en rien la composition originalement prévue. Ainsi, les deux sièges désignés pour la communauté autochtone, mais qui sont a ce jour vacants, demeureront réservés.

(13) Statistiques compilées par la SERPO.

(14) Fabrique d'Oka, Sociéte d'histoire d'Oka. Chervalier de Colomb, CDE Deux-Montagnes, etc

(15) Ces prévisions sont basées sur les sommes présentement réinvesties et sur les profits antici. pés pour l'anné tiscale qui se termine le 31 mars 1995.

(16) Ce déficit a été enregistré à cause des maurvaises conditions climatiques del'śté 1992 et d'un manque à gagner lié au fait que l'exploitation services alimentaires avait été cédée à un concessionnaire avant l'arrivee de la SERPO. II faut également noter que la Corporation ne bénéticiè pas de subwentions. 